\title{
PRIME DIVISORS OF IRREDUCIBLE CHARACTER DEGREES
}

\author{
HUNG P. TONG-VIET
}

\begin{abstract}
Let $G$ be a finite group. We denote by $\rho(G)$ the set of primes which divide some character degrees of $G$ and by $\sigma(G)$ the largest number of distinct primes which divide a single character degree of $G$. We show that $|\rho(G)| \leq 2 \sigma(G)+1$ when $G$ is an almost simple group. For arbitrary finite groups $G$, we show that $|\rho(G)| \leq 2 \sigma(G)+1$ provided that $\sigma(G) \leq 2$.
\end{abstract}

1. Introduction. Throughout this paper, all groups are finite, and all characters are complex characters. The set of all complex irreducible characters of $G$ is denoted by $\operatorname{Irr}(G)$, and we let $\operatorname{cd}(G)$ be the set of all complex irreducible character degrees of $G$. We define $\rho(G)$ to be the set of primes which divide some character degree of $G$. For $\chi \in \operatorname{Irr}(G)$, let $\pi(\chi)$ be the set of all prime divisors of $\chi(1)$, and let $\sigma(\chi)=|\pi(\chi)|$. Moreover, $\sigma(G)$ is defined to be the maximum value of $\sigma(\chi)$ when $\chi$ runs over the set $\operatorname{Irr}(G)$. Huppert's $\rho-\sigma$ conjecture proposed by Huppert in [7] states that if $G$ is a solvable group, then $|\rho(G)| \leq 2 \sigma(G)$; and, if $G$ is an arbitrary group, then $|\rho(G)| \leq 3 \sigma(G)$. For solvable groups, this conjecture has been verified by Manz [11] and Gluck [6] when $\sigma(G)=1$ and 2, respectively. In general, it is proved by Manz and Wolf [13] that $|\rho(G)| \leq 3 \sigma(G)+2$. For arbitrary groups, Manz [12] showed that $|\rho(G)|=3$ if $G$ is nonsolvable and $\sigma(G)=1$. Recently, it has been proved by Casolo and Dolfi [3] that $|\rho(G)| \leq 7 \sigma(G)$ for any arbitrary groups $G$. In [13], Manz and Wolf proposed that, for any group $G$,

$$
|\rho(G)| \leq 2 \sigma(G)+1 .
$$

We call this new conjecture the strengthened Huppert's $\rho-\sigma$ conjecture. Obviously, this new conjecture is stronger than the original one. In

2010 AMS Mathematics subject classification. Primary $20 \mathrm{C} 15$.

Keywords and phrases. Character degrees, Huppert's $\rho-\sigma$ conjecture.

Received by the editors on March 11, 2013, and in revised form on August 19, 2013. 
this paper, we first improve the result due to Alvis and Barry in [1] by proving the following.

Theorem A. Let $G$ be an almost simple group. Then $|\rho(G)| \leq 2 \sigma(G)$ unless $G \cong \mathrm{PSL}_{2}\left(2^{f}\right)$ with $f \geq 2$ and $\left|\pi\left(2^{f}-1\right)\right|=\left|\pi\left(2^{f}+1\right)\right|$. For the exceptions, we have $|\rho(G)|=2 \sigma(G)+1$.

This verifies the strengthened Huppert's $\rho-\sigma$ conjecture for almost simple groups. In the next theorem, we verify this new conjecture for groups $G$ with $\sigma(G) \leq 2$.

Theorem B. Let $G$ be a finite group. If $\sigma(G) \leq 2$, then $|\rho(G)| \leq$ $2 \sigma(G)+1$.

Notice that Theorem B is also a generalization to $[\mathbf{1 9}$, Theorem A].

Notation. For a positive integer $n$, we denote the set of all prime divisors of $n$ by $\pi(n)$. If $G$ is a group, then we write $\pi(G)$ instead of $\pi(|G|)$ for the set of all prime divisors of the order of $G$. If $N \unlhd G$ and $\theta \in \operatorname{Irr}(N)$, then the inertia group of $\theta$ in $G$ is denoted by $I_{G}(\theta)$. We write $\operatorname{Irr}(G \mid \theta)$ for the set of all irreducible constituents of $\theta^{G}$. Moreover, if $\chi \in \operatorname{Irr}(G)$, then $\operatorname{Irr}\left(\chi_{N}\right)$ is the set of all irreducible constituents of $\chi$ when restricted to $N$. Recall that a group $G$ is said to be an almost simple group with socle $S$ if there exists a nonabelian simple group $S$ such that $S \unlhd G \leq \operatorname{Aut}(S)$. The greatest common divisor of two integers $a$ and $b$ is $\operatorname{gcd}(a, b)$. Denote by $\Phi_{k}:=\Phi_{k}(q)$ the value of the $k$ th cyclotomic polynomial evaluated at $q$. Other notation is standard.

2. Proof of Theorem A. If $G$ is an almost simple group, then $G$ has no normal abelian Sylow subgroup and so, by Ito-Michler's theorem [14, Theorem 5.4], $\rho(G)=\pi(G)$. This fact will be used without any further reference.

Lemma 2.1. Let $S$ be a sporadic simple group, the Tits group or an alternating group of degree at least 7 . If $G$ is an almost simple group with socle $S$, then

$$
|\pi(G)|=|\pi(S)| \leq 2 \sigma(G) .
$$


Proof. Observe first that, if $S$ is one of the simple groups in the lemma, and $G$ is any almost simple group with socle $S$, then $\pi(G)=$ $\pi(S)$. Since $S \unlhd G$, we see that $\sigma(S) \leq \sigma(G)$. Thus, it suffices to show that $|\pi(S)| \leq 2 \sigma(S)$. By using [4], we can easily check that $|\pi(S)| \leq 2 \sigma(S)$ when $S$ is a sporadic simple group, the Tits group or an alternating group of degree $n$ with $7 \leq n \leq 14$. Finally, if $S \cong \mathrm{A}_{n}$ with $n \geq 15$, then the result in [2] yields that $|\pi(S)|=\sigma(S)$. This completes the proof.

For $\epsilon= \pm$, we use the convention that $\operatorname{PSL}_{n}^{\epsilon}(q)$ is $\operatorname{PSL}_{n}(q)$ if $\epsilon=+$ and $\operatorname{PSU}_{n}(q)$ if $\epsilon=-$. Let $q \geq 2$ and $n \geq 3$ be integers with $(n, q) \neq(6,2)$. A prime $\ell$ is called a primitive prime divisor of $q^{n}-1$ if $\ell \mid q^{n}-1$ but $\ell \nmid q^{m}-1$ for any $m<n$. By Zsigmondy's theorem [21], the primitive prime divisors of $q^{n}-1$ always exist. We denote by $\ell_{n}(q)$ the smallest primitive prime divisor of $q^{n}-1$. In Table 1 , which is taken from [10], we give the orders of two maximal tori $T_{i}$ and the corresponding two primitive prime divisors $\ell_{i}$, for $i=1,2$, of classical groups.

Let $\mathcal{C}$ be the set consisting of the following simple groups:

$$
\begin{array}{lllll}
\operatorname{PSL}_{2}(q), & \operatorname{PSL}_{3}(q), & \operatorname{PSU}_{3}(q), & \operatorname{PSp}_{4}(q) & \operatorname{PSL}_{4}(2), \\
\operatorname{PSL}_{6}(2), & \operatorname{PSL}_{7}(2), & \operatorname{PSU}_{4}(2), & \operatorname{PSU}_{4}(3), & \operatorname{PSU}_{6}(2), \\
\mathrm{Sp}_{4}(2)^{\prime}, & \operatorname{Sp}_{6}(2), & \operatorname{Sp}_{8}(2), & \Omega_{7}(3), & \Omega_{8}^{+}(2), \\
\Omega_{8}^{-}(2), & { }^{3} \mathrm{D}_{4}(2), & \mathrm{G}_{2}(2)^{\prime}, & \mathrm{G}_{2}(3), & \mathrm{G}_{2}(4) .
\end{array}
$$

Lemma 2.2. Let $S$ be a finite simple group of Lie type in characteristic $p$ which is not the Tits groups nor $\operatorname{PSL}_{2}\left(2^{f}\right)$ with $f \geq 2$. Then $|\pi(S)| \leq 2 \sigma(S)$.

Proof. We consider the following cases.

Case 1. $S \cong \operatorname{PSL}_{2}(q)$, where $q=p^{f} \geq 5$ is odd.

Since $\mathrm{PSL}_{2}(5) \cong \mathrm{PSL}_{2}(4)$, we can assume that $q>5$. In this case, all character degrees of $S$ divide $q, q-1$ or $q+1$. Observe that

$$
\pi(S)=\{p\} \cup \pi(q-1) \cup \pi(q+1),\{p\} \cap \pi(q \pm 1)=\emptyset
$$

and

$$
\pi(q-1) \cap \pi(q+1)=\{2\} .
$$


TABLE 1. Two tori for classical groups.

\begin{tabular}{|c|c|c|c|c|}
\hline$G=G(q)$ & $\left|T_{1}\right|$ & $\left|T_{2}\right|$ & $\ell_{1}$ & $\ell_{2}$ \\
\hline $\mathrm{A}_{n}$ & $\begin{array}{l}\left(q^{n+1}-1\right) / \\
(q-1)\end{array}$ & $q^{n}-1$ & $\ell_{n+1}(q)$ & $\ell_{n}(q)$ \\
\hline $\begin{array}{l}{ }^{2} \mathrm{~A}_{n}, \\
(n \equiv 0(4))\end{array}$ & $\begin{array}{l}\left(q^{n+1}+1\right) / \\
(q+1)\end{array}$ & $q^{n}-1$ & $\ell_{2 n+2}(q)$ & $\ell_{n}(q)$ \\
\hline${ }^{2} \mathrm{~A}_{n}$ & $\begin{array}{l}\left(q^{n+1}-1\right) / \\
(q+1)\end{array}$ & $q^{n}+1$ & $\ell_{(n+1) / 2}(q)$ & $\ell_{2 n}(q)$ \\
\hline $\begin{array}{l}(n \equiv 1(4)) \\
{ }^{2} \mathrm{~A}_{n}\end{array}$ & $\begin{array}{l}\left(q^{n+1}+1\right) / \\
(q+1)\end{array}$ & $q^{n}-1$ & $\ell_{2 n+2}(q)$ & $\ell_{n / 2}(q)$ \\
\hline $\begin{array}{l}(n \equiv 2(4)) \\
{ }^{2} \mathrm{~A}_{n},\end{array}$ & $\begin{array}{l}\left(q^{n+1}-1\right) / \\
(q+1)\end{array}$ & $q^{n}+1$ & $\ell_{n+1}(q)$ & $\ell_{2 n}(q)$ \\
\hline$(n \equiv 3(4))$ & & & & \\
\hline $\begin{array}{l}\mathrm{B}_{n}, \mathrm{C}_{n} \\
(n \geq 3 \text { odd })\end{array}$ & $q^{n}+1$ & $q^{n}-1$ & $\ell_{2 n}(q)$ & $\ell_{n}(q)$ \\
\hline $\begin{array}{l}\mathrm{B}_{n}, \mathrm{C}_{n} \\
(n \geq 2 \text { even })\end{array}$ & $q^{n}+1$ & $\left(q^{n-1}+1\right)(q+1)$ & $\ell_{2 n}(q)$ & $\ell_{2 n-2}(q)$ \\
\hline $\begin{array}{l}\mathrm{D}_{n} \\
(n \geq 5 \text { odd })\end{array}$ & $\left(q^{n-1}+1\right)(q+1)$ & $q^{n}-1$ & $\ell_{2 n-2}(q)$ & $\ell_{n}(q)$ \\
\hline $\begin{array}{l}\mathrm{D}_{n} \\
(n \geq 4 \text { even })\end{array}$ & $\left(q^{n-1}+1\right)(q+1)$ & $\left(q^{n-1}-1\right)(q-1)$ & $\ell_{2 n-2}(q)$ & $\ell_{n-1}(q)$ \\
\hline${ }^{2} \mathrm{D}_{n}$ & $q^{n}+1$ & $\left(q^{n-1}+1\right)(q-1)$ & $\ell_{2 n}(q)$ & $\ell_{2 n-2}(q)$ \\
\hline
\end{tabular}

Hence, we obtain that

$$
\begin{aligned}
|\pi(S)| & =1+\sigma(q+1)+\sigma(q-1)-|\pi(q-1) \cap \pi(q+1)| \\
& =\sigma(q+1)+\sigma(q-1) \leq 2 \sigma(S) .
\end{aligned}
$$

Case 2. $S \cong \operatorname{PSL}_{3}^{\epsilon}(q)$ with $q=p^{f}$ and $\epsilon= \pm$. $\operatorname{As}_{\mathrm{PSL}_{3}}(2) \cong$ $\mathrm{PSL}_{2}(7)$ and $\mathrm{PSU}_{3}(2)$ are not simple, we can assume that $q>2$. The cases when $q=3$ or 4 can be checked directly using [4]. So, we can assume that $q \geq 5$. By [17], $S$ possesses irreducible characters $\chi_{i}$, $i=1,2$, with degree

$$
\chi_{1}(1)=(q-\epsilon 1)^{2}(q+\epsilon 1) \quad \text { and } \quad \chi_{2}(1)=q\left(q^{2}+\epsilon q+1\right) .
$$


Let $d=\operatorname{gcd}(3, q-\epsilon 1)$. Then

$$
|S|=\frac{1}{d} q^{3}\left(q^{2}-1\right)\left(q^{3}-\epsilon 1\right)=\frac{1}{d} q^{3}(q-\epsilon 1)^{2}(q+\epsilon 1)\left(q^{2}+\epsilon q+1\right),
$$

and so

$$
\pi(S)=\pi\left(\chi_{1}\right) \cup \pi\left(\chi_{2}\right) .
$$

Therefore, $|\pi(S)| \leq 2 \sigma(S)$ as wanted.

Case 3. $S \cong \operatorname{PSp}_{4}(q)$ with $q=p^{f}>2$.

By $[\mathbf{5}, \mathbf{1 8}], S$ has two irreducible characters $\chi_{i}, i=1,2$, with degrees $\Phi_{1}^{2} \Phi_{2}^{2}$ and $q \Phi_{1} \Phi_{4}$, respectively. Since

$$
|S|=\frac{1}{d} q^{4} \Phi_{1}^{2} \Phi_{2}^{2} \Phi_{4}
$$

where $d=\operatorname{gcd}(2, q-1)$, we deduce that

$$
\pi(S)=\pi\left(\chi_{1}\right) \cup \pi\left(\chi_{2}\right),
$$

and thus $|\pi(S)| \leq 2 \sigma(S)$.

Case 4. $S$ is one of the remaining simple groups in the list $\mathcal{C}$.

Using [4], it is routine to check that $|\pi(S)| \leq 2 \sigma(S)$ in all these cases.

Case 5. $S$ is not in the list $\mathcal{C}$.

We consider the following setup. Let $\mathscr{G}$ be a simple, simply connected algebraic group defined over a field of size $q$ in characteristic $p$, and let $F$ be a Frobenius map on $\mathscr{G}$ such that $S \cong L / Z$, where $L:=\mathscr{G}^{F}$ and $Z:=\mathrm{Z}(L)$. Let the pair $\left(\mathscr{G}^{*}, F^{*}\right)$ be dual to $(\mathscr{G}, F)$ and let $L^{*}:=\mathscr{G}^{* F^{*}}$. By Lusztig's theory, the irreducible characters of $\mathscr{G}^{F}$ are partitioned into rational series $\mathscr{E}\left(\mathscr{G}^{F},(s)\right)$ which are indexed by $\left(\mathscr{G}^{* F^{*}}\right)$-conjugacy classes $(s)$ of semisimple elements $s \in \mathscr{G}^{* F^{*}}$. Furthermore, if $\operatorname{gcd}(|s|,|Z|)=1$, then every $\chi \in \mathscr{E}\left(\mathscr{G}^{F},\left(s_{i}\right)\right)$ is trivial at $Z$, and thus $\chi \in \operatorname{Irr}(S)=\operatorname{Irr}(L / Z)$. (See [15, page 349]). Notice also that $\chi(1)$ is divisible by $\left|L^{*}: \mathbf{C}_{L^{*}}(s)\right|_{p^{\prime}}$.

For simple classical groups of Lie type, the restriction on $S$ guarantees that both primitive prime divisors $\ell_{i}$ in Table 1 exist. Let $s_{i} \in \mathscr{G}^{* F^{*}}$ with $\left|s_{i}\right|=\ell_{i}, i=1,2$. Then $\mathbf{C}_{L^{*}}\left(s_{i}\right)=T_{i}$ for $i=1,2$, 
where $T_{i}$ are maximal tori of $L^{*}$. Similarly, for each simple exceptional group of Lie type $S$, by [15, Lemma 2.3], one can find two semisimple elements $s_{i} \in \mathscr{G}^{*} F^{*}$ with $\left|s_{i}\right|=\ell_{i}, i=1,2$. In both cases, we have that $\left(\ell_{i},|Z|\right)=1$ for $i=1,2$ and, if $a:=\operatorname{gcd}\left(\left|\mathbf{C}_{L^{*}}\left(s_{1}\right)\right|,\left|\mathbf{C}_{L^{*}}\left(s_{2}\right)\right|\right)$, then either $a=1$ or, if a prime $r$ divides $a$, then $r$ also divides $\left|L^{*}: \mathbf{C}_{L^{*}}\left(s_{i}\right)\right|_{p^{\prime}}$ for some $i$. Let $\chi_{i} \in \mathscr{E}\left(\mathscr{G}^{F},\left(s_{i}\right)\right), i=1,2$, be such that $\chi_{i}(1)=\left|L^{*}: \mathbf{C}_{L^{*}}\left(s_{i}\right)\right|_{p^{\prime}}$. Then $\chi_{i} \in \operatorname{Irr}(S)$ for $i=1,2$ and

$$
\pi(S)=\{p\} \cup \pi\left(\chi_{1}\right) \cup \pi\left(\chi_{2}\right) .
$$

Notice that $p$ is relatively prime to both $\chi_{i}(1)$ for $i=1,2$. So,

$$
\begin{aligned}
|\pi(S)| & =\left|\{p\} \cup \pi\left(\chi_{1}\right) \cup \pi\left(\chi_{2}\right)\right| \\
& =1+\left|\pi\left(\chi_{1}\right)\right|+\left|\pi\left(\chi_{2}\right)\right|-\left|\pi\left(\chi_{1}\right) \cap \pi\left(\chi_{2}\right)\right| \\
& =\sigma\left(\chi_{1}\right)+\sigma\left(\chi_{2}\right)-\left(\left|\pi\left(\chi_{1}\right) \cap \pi\left(\chi_{2}\right)\right|-1\right) \\
& \leq 2 \sigma(S)-\left(\left|\pi\left(\chi_{1}\right) \cap \pi\left(\chi_{2}\right)\right|-1\right) .
\end{aligned}
$$

If we can show that $\left|\pi\left(\chi_{1}\right) \cap \pi\left(\chi_{2}\right)\right| \geq 1$, then clearly $|\pi(S)| \leq 2 \sigma(S)$, and we are done. By way of contradiction, assume that $\pi\left(\chi_{1}\right) \cap \pi\left(\chi_{2}\right)$ is empty. Then $\operatorname{gcd}\left(\chi_{1}(1), \chi_{2}(1)\right)=1$, and so

$$
\operatorname{gcd}\left(\left|L^{*}: \mathbf{C}_{L^{*}}\left(s_{1}\right)\right|_{p^{\prime}},\left|L^{*}: \mathbf{C}_{L^{*}}\left(s_{2}\right)\right|_{p^{\prime}}\right)=1 .
$$

It follows that $\left|L^{*}\right|_{p^{\prime}}$ must divide $\left|\mathbf{C}_{L^{*}}\left(s_{1}\right)\right|_{p^{\prime}} \cdot\left|\mathbf{C}_{L^{*}}\left(s_{2}\right)\right|_{p^{\prime}}$. However, we can check by using [15, Lemma 2.3] and Table 1 that this is not the case. The proof is now complete.

We now prove Theorem A which we restate here.

Theorem 2.3. Let $G$ be an almost simple group. Then $|\rho(G)| \leq 2 \sigma(G)$ unless $G \cong \mathrm{PSL}_{2}\left(2^{f}\right)$ with $\left|\pi\left(2^{f}-1\right)\right|=\left|\pi\left(2^{f}+1\right)\right|$. For the exceptions, we have $|\rho(G)|=2 \sigma(G)+1$.

Proof. Let $G$ be an almost simple group with simple socle $S$. Since $S \unlhd G$, we obtain that $\sigma(S) \leq \sigma(G)$.

Case 1. $S \cong \mathrm{PSL}_{2}(q)$ with $q=2^{f} \geq 4$.

It is well known that $|S|=q\left(q^{2}-1\right), \operatorname{gcd}\left(2^{f}-1,2^{f}+1\right)=1$ and

$$
\operatorname{cd}(S)=\{1, q-1, q, q+1\} .
$$


If $|\pi(q-1)|=|\pi(q+1)|$, then

$$
\pi(S)=\{2\} \cup \pi(q-1) \cup \pi(q+1),
$$

and thus $|\pi(S)|=2 \sigma(S)+1$ as $\sigma(S)=\left|\pi\left(2^{f} \pm 1\right)\right|$. Assume that $|\pi(q-1)| \neq|\pi(q+1)|$. Then $\left|\pi\left(2^{f}+\delta\right)\right|>\left|\pi\left(2^{f}-\delta\right)\right|$ for some $\delta \in\{ \pm 1\}$. Hence, $\sigma(S)=\left|\pi\left(2^{f}+\delta\right)\right|$, and thus

$$
\begin{aligned}
|\pi(S)| & =\left|\{2\} \cup \pi\left(2^{f}-\delta\right) \cup \pi\left(2^{f}+\delta\right)\right| \\
& =1+\left|\pi\left(2^{f}-\delta\right)\right|+\left|\pi\left(2^{f}+\delta\right)\right| .
\end{aligned}
$$

Since $\left|\pi\left(2^{f}+\delta\right)\right| \geq\left|\pi\left(2^{f}-\delta\right)\right|+1$, we obtain that

$$
|\rho(S)| \leq 2\left|\pi\left(2^{f}+\delta\right)\right| \leq 2 \sigma(S) .
$$

Thus, the result holds when $G=S$.

Assume now that $|G: S|$ is nontrivial. We know that $\operatorname{Aut}(S)=$ $S \cdot\langle\varphi\rangle$, where $\varphi$ is a field automorphism of $S$ of order $f$. Thus, $G=S \cdot\langle\psi\rangle$, with $\psi \in\langle\varphi\rangle$. If $f=2$, then $G \cong \mathrm{A}_{5} \cdot 2$, and obviously $|\pi(G)| \leq 2 \sigma(G)$. Hence, we can assume that $f>2$. Clearly, if $f \equiv 2(\bmod 4)$ and $G=S \cdot\langle\varphi\rangle$, then $|G: S|>2$. So by $[\mathbf{2 0}$, Theorem A], $G$ has two irreducible characters $\chi_{i} \in \operatorname{Irr}(G), i=1,2$, with $\chi_{1}(1)=|G: S|(q-1)$ and $\chi_{2}(1)=|G: S|(q+1)$. Obviously,

$$
\pi(G)=\{2\} \cup \pi\left(\chi_{1}\right) \cup \pi\left(\chi_{2}\right)
$$

and

$$
\pi\left(\chi_{1}\right) \cap \pi\left(\chi_{2}\right)=\pi(|G: S|) \neq \emptyset .
$$

If $|G: S|$ is even, then

$$
|\rho(G)|=\left|\pi\left(\chi_{1}\right) \cup \pi\left(\chi_{2}\right)\right| \leq\left|\pi\left(\chi_{1}\right)\right|+\left|\pi\left(\chi_{2}\right)\right| \leq 2 \sigma(G) .
$$

If $|G: S|$ is odd, then

$$
\begin{aligned}
|\rho(G)| & =\left|\{2\} \cup \pi\left(\chi_{1}\right) \cup \pi\left(\chi_{2}\right)\right| \\
& =1+\left|\pi\left(\chi_{1}\right)\right|+\left|\pi\left(\chi_{2}\right)\right|-\left|\pi\left(\chi_{1}\right) \cap \pi\left(\chi_{2}\right)\right| \\
& =\sigma\left(\chi_{1}\right)+\sigma\left(\chi_{2}\right)-(|\pi(|G: S|)|-1) \\
& \leq \sigma\left(\chi_{1}\right)+\sigma\left(\chi_{2}\right) \\
& \leq 2 \sigma(G) .
\end{aligned}
$$


Case 2. $S$ is a sporadic simple group, the Tits group or an alternating group of degree at least 7 .

By Lemma 2.1, we obtain that $|\rho(G)| \leq 2 \sigma(G)$.

Case 3. $S$ is a finite simple group of Lie type in characteristic $p$ and $S$ is not the Tits group nor $\operatorname{PSL}_{2}\left(2^{f}\right)$ with $f \geq 2$.

Subcase 3a. $\pi(G)=\pi(S)$.

By Lemma 2.2, we have that $|\pi(S)| \leq 2 \sigma(S)$. Thus,

$$
|\rho(G)|=|\pi(S)| \leq 2 \sigma(S) \leq 2 \sigma(G) .
$$

Subcase 3b. $\pi:=\pi(G)-\pi(S)$ is nonempty.

Let $A$ be the subgroup of the group of coprime outer automorphisms of $S$ induced by the action of $G$ on $S$. By [15, Lemma 2.10], $A$ is cyclic and central in $\operatorname{Out}(S)$. Moreover, $A$ is generated by a fixed field automorphism $\gamma \in \operatorname{Out}(S)$. It follows that the group $S \cdot A$ is normal in $G$ and $\pi(S \cdot A)=\pi(G)$. Thus we can assume that $G=S \cdot A$ with $A=\langle\gamma\rangle$ and $\gamma$ a field automorphism of $S$. Furthermore, $\pi(\gamma)=\pi$. Replacing $A$ by a normal subgroup if necessary, we can also assume that $|A|=|\gamma|$ is the product of all distinct primes in $\pi$.

As in the proof of Lemma 2.2 , let $\mathscr{G}$ be a simple, simply connected algebraic group defined over a field of size $q=p^{f}$ in characteristic $p$, and let $F$ be a Frobenius map of $\mathscr{G}$ such that $S \cong L / Z$, where $L:=\mathscr{G}^{F}$ and $Z:=\mathrm{Z}(L)$. Let the pair $\left(\mathscr{G}^{*}, F^{*}\right)$ be dual to $(\mathscr{G}, F)$, and let $L^{*}:=\mathscr{G}^{*} F^{*}$. As $\pi \subseteq \pi(f)$, where $\pi=\pi(G)-\pi(S)$, it is easy to check that both the primitive prime divisors in [15, Lemmas 2.3 and 2.4] exist, and thus one can find two semisimple elements $s_{i} \in \mathscr{G}^{*} F^{*}$ with $\left|s_{i}\right|=\ell_{i}$ such that $\left(\ell_{i},|Z|\right)=1$ for $i=1,2$. Arguing as in the proof of Lemma 2.2, we obtain that

$$
\pi(S)=\{p\} \cup \pi\left(\chi_{1}\right) \cup \pi\left(\chi_{2}\right),
$$

where $\chi_{i} \in \mathscr{E}\left(\mathscr{G}^{F},\left(s_{i}\right)\right)$ such that $\chi_{i}(1)=\left|L^{*}: \mathbf{C}_{L^{*}}\left(s_{i}\right)\right|_{p^{\prime}}$ and $\chi_{i}$ can be considered as characters of $S$ for $i=1,2$.

We next claim that the inertia group for both $\chi_{i}, i=1,2$, in $G$ is exactly $S$. It suffices to show that no field automorphism of $S$ of 
prime order can fix $\chi_{i}$ for $i=1,2$. Let $\tau$ be a field automorphism of $S$ of prime order $s$. We can extend $\tau$ to an automorphism of $\mathscr{G}^{F}$ and $\mathscr{G}^{* F^{*}}$, which we also denote by $\tau$. Notice that $\mathbf{C}_{\mathscr{G} * F^{*}}(\tau)$ is a finite group of Lie type of the same type as that of $\mathscr{G}^{* F^{*}}$ but defined over a field of size $q^{1 / s}$. Now it is straightforward to check that both $\ell_{i}, i=1,2$, are relatively prime to $\left|\mathbf{C}_{\mathscr{G} * F^{*}}(\tau)\right|$. Hence, $\mathscr{G}^{*} F^{*}$ conjugacy classes $\left(s_{i}\right)$ of $s_{i}$ in $\mathscr{G}^{* F^{*}}$ are not $\tau$-invariant for $i=1,2$ (see [15, Proposition 2.6]). Then $\tau\left(s_{i}\right)$ and $s_{i}$ are not $\mathscr{G}^{* F^{*}}$-conjugate for $i=1,2$, and thus $\chi_{i} \in \mathscr{E}\left(\mathscr{G}^{F},\left(s_{i}\right)\right), i=1,2$, are not $\tau$-invariant (see [15, Theorem 2.7]). Therefore, we obtain that $\chi_{i}^{G} \in \operatorname{Irr}(G)$ for $i=1,2$; hence, $\chi_{i}^{G}(1)=|G: S| \chi_{i}(1) \in \operatorname{cd}(G)$. Since

$$
\pi(S)=\{p\} \cup \pi\left(\chi_{1}\right) \cup \pi\left(\chi_{2}\right) \text { and } \pi(G)=\pi(S) \cup \pi(|G: S|),
$$

we obtain that

$$
\begin{aligned}
\pi(G) & =\{p\} \cup \pi\left(|G: S| \chi_{1}(1)\right) \cup \pi\left(|G: S| \chi_{2}(1)\right) \\
& =\{p\} \cup \pi\left(\chi_{1}^{G}\right) \cup \pi\left(\chi_{2}^{G}\right) .
\end{aligned}
$$

Moreover, $p \nmid|G: S| \chi_{i}(1)=\chi_{i}^{G}(1)$ for $i=1,2$, and

$$
\left|\pi\left(\chi_{1}^{G}\right) \cap \pi\left(\chi_{2}^{G}\right)\right| \geq 1 .
$$

Therefore,

$$
\begin{aligned}
|\pi(G)| & =1+\sigma\left(\chi_{1}^{G}\right)+\sigma\left(\chi_{2}^{G}\right)-\left|\pi\left(\chi_{1}^{G}\right) \cap \pi\left(\chi_{2}^{G}\right)\right| \\
& \leq 2 \sigma(G)-\left(\left|\pi\left(\chi_{1}^{G}\right) \cap \pi\left(\chi_{2}^{G}\right)\right|-1\right) \\
& \leq 2 \sigma(G) .
\end{aligned}
$$

The proof is now complete.

The next results will be needed in the proof of Theorem B.

Lemma 2.4. Let $S$ be a nonabelian simple group. If $\sigma(S) \leq 2$, then $S$ is one of the following groups.

(i) $S \cong \mathrm{PSL}_{2}\left(2^{f}\right)$ with $\left|\pi\left(2^{f} \pm 1\right)\right| \leq 2$, and so $|\pi(S)| \leq 5$.

(ii) $S \cong \mathrm{PSL}_{2}(q)$ with $q>5$ odd and $|\pi(q \pm 1)| \leq 2$ and so $|\pi(S)| \leq 4$.

(iii) $S \in\left\{\mathrm{M}_{11}, \mathrm{~A}_{7},{ }^{2} \mathrm{~B}_{2}(8),{ }^{2} \mathrm{~B}_{2}(32), \mathrm{PSL}_{3}^{ \pm}(3), \mathrm{PSL}_{3}^{ \pm}(4), \mathrm{PSL}_{3}(8)\right\}$ and $|\pi(S)|=4$. 
Proof. As $S$ is a nonabelian simple group, we have that $|\pi(S)| \geq 3$. If $S \cong \operatorname{PSL}_{2}(q)$ with $q \geq 4$, then the lemma follows easily as the character degree set of $S$ is known. Now assume that $S \nsubseteq \operatorname{PSL}_{2}(q)$. Then Lemmas 2.2 and 2.1 imply that $|\pi(S)| \leq 2 \sigma(S)$. So, $3 \leq|\pi(S)| \leq 4$. By checking the list of nonabelian simple groups with at most four prime divisors in [8], we deduce that only those nonabelian simple groups appearing in (iii) above satisfy the hypotheses of the lemma.

Lemma 2.5. Let $G$ be an almost simple group with simple socle $S$. If $\sigma(G) \leq 2$, then $\pi(G)=\pi(S)$, where $S$ is one of the simple groups in Lemma 2.4.

Proof. Since $\sigma(S) \leq \sigma(G) \leq 2$, we deduce that $S$ is isomorphic to one of the simple groups in the conclusion of Lemma 2.4. If $|\pi(S)|=3$, then $S$ is one of the simple groups in [8, Table 1], and we can check that $\pi(G)=\pi(S)$ in these cases. Thus, we assume that $|\pi(S)| \geq 4$. Now, if $G=S$, then we have nothing to prove. So, we assume that $G \neq S$. In particular, $G \nRightarrow \operatorname{PSL}_{2}\left(2^{f}\right)$ with $f \geq 2$. Then $|\pi(G)| \leq 2 \sigma(G) \leq 4$ by Theorem A, and thus $4 \leq|\pi(S)| \leq|\pi(G)| \leq 4$, which forces $|\pi(S)|=|\pi(G)|$ and, hence, $\pi(G)=\pi(S)$ as wanted.

3. Proof of Theorem B. The following two lemmas are obvious.

Lemma 3.1. Let $A$ and $B$ be groups such that $|\rho(A)| \geq 3$ and $|\rho(B)| \geq 3$. If

$$
\sigma(A \times B) \leq 2
$$

then $\sigma(A)=1=\sigma(B)$.

Lemma 3.2. Let $N$ be a normal subgroup of a group $G$. If $\rho(G / N)=$ $\pi(G / N)$, then

$$
\rho(G)-\rho(G / N) \subseteq \rho(N)
$$

Recall that the solvable radical of a group $G$ is the largest normal solvable subgroup of $G$.

Lemma 3.3. Let $G$ be a nonsolvable group, and let $N$ be the solvable radical of $G$. Suppose that $\sigma(G) \leq 2$ and $|\rho(G)| \geq 5$. Then $G / N$ is an almost simple group. 
Proof. We first claim that, if $M / N$ is a chief factor of $G$, then $M / N$ is a nonabelian simple group.

Let $M$ be a normal subgroup of $G$ such that $M / N$ is a chief factor of $G$. Since $N$ is the largest normal solvable subgroup of $G$, we deduce that $M / N$ is nonsolvable so that $M / N \cong S^{k}$ for some integer $k \geq 1$ and some nonabelian simple group $S$. Let $C / N=\mathbf{C}_{G / N}(M / N)$. Then $G / C$ embeds into $\operatorname{Aut}\left(S^{k}\right)$.

Assume first that $k \geq 3$. Since $|\rho(S)|=|\pi(S)| \geq 3$, there exist three distinct prime divisors $r_{i}, 1 \leq i \leq 3$, and three characters $\psi_{i} \in \operatorname{Irr}(S)$ for $1 \leq i \leq 3$ with $r_{i} \mid \psi_{i}(1)$. Let

$$
\varphi=\psi_{1} \times \psi_{2} \times \psi_{3} \times 1 \times \cdots \times 1 \in \operatorname{Irr}\left(S^{k}\right) .
$$

Then $\sigma(\varphi) \geq 3$, which is a contradiction since

$$
\sigma\left(S^{k}\right)=\sigma(M / N) \leq \sigma(M) \leq \sigma(G) \leq 2 .
$$

Thus $k \leq 2$.

Now assume that $k=2$. Let $B / C=(G / C) \cap \operatorname{Aut}(S)^{2}$. Then $G / B$ is a nontrivial subgroup of the symmetric group of degree 2 , and thus $|G: B|=2$. Since $S^{2} \cong M C / C \unlhd B / C \unlhd G / C$ and $\sigma(G) \leq 2$, we deduce that $\sigma\left(S^{2}\right) \leq 2$, and thus $\sigma(S)=1$, by Lemma 3.1. By [12, Satz 8], we know that $S$ is isomorphic to either $\mathrm{PSL}_{2}(4)$ or $\mathrm{PSL}_{2}(8)$. In both cases, we obtain that $\pi(\operatorname{Aut}(S))=\pi(S)$; hence, $\pi(B / C)=\pi(S)$. Moreover, as $|G: B|=2$, we deduce that $\pi(G / C)=\pi(S)$. As $G / C$ has no nontrivial normal abelian Sylow subgroups, Ito-Michler's theorem yields that $\rho(G / C)=\pi(G / C)=\pi(S)$. Since $|\pi(G / C)|=|\pi(S)|=3$ and $|\rho(G)| \geq 5$, there exists $r \in \rho(G)-\pi(G / C)$. Then $r>2$ and $r \in \rho(C)$ by Lemma 3.2. Let $\theta \in \operatorname{Irr}(C)$ be such that $r \mid \theta(1)$. Let $L$ be a normal subgroup of $M C$ such that $L / C \cong S$. Notice that $M C / C \cong S^{2}$. By applying [19, Lemma 4.2], $\theta$ extends to $\theta_{0} \in \operatorname{Irr}(L)$. By Gallagher's theorem [9, Corollary 6.17], $\theta_{0} \mu \in \operatorname{Irr}(L)$ for all $\mu \in \operatorname{Irr}(L / C)$. Let $\mu_{0} \in \operatorname{Irr}(L / C)$ with $2 \mid \mu_{0}(1)$, and let $\varphi=\theta_{0} \mu_{0} \in \operatorname{Irr}(L)$. Then $\pi(\varphi(1))=\{2, r\}$ with $r>2$. As $M C / L \cong S$, we can apply [19, Lemma 4.2] again to obtain that $\varphi$ extends to $\varphi_{0} \in \operatorname{Irr}(M C)$ and then, by applying Gallagher's theorem, $\varphi_{0} \mu \in \operatorname{Irr}(M C)$ for all $\mu \in \operatorname{Irr}(M C / L)$. Clearly, $M C / L \cong S$ has an irreducible character $\tau \in \operatorname{Irr}(M C / L)$ with $s \mid \tau(1)$, where $s \notin\{2, r\}$. We now have that $\varphi_{0} \tau \in \operatorname{Irr}(M C)$. But then this is a contradiction 
as $\pi\left(\varphi_{0}(1) \tau(1)\right)=\{2, s, r\}$. This contradiction shows that $k=1$, as wanted.

Let $M / N$ be a chief factor of $G$, and let $C / N=\mathbf{C}_{G / N}(M / N)$. We claim that $C=N$ and thus $G / N$ is an almost simple group as required. By the claim above, we know that $M / N \cong S$ for some nonabelian simple group $S$. Hence, $G / C$ is an almost simple group with socle $M C / C \cong M / N$. Suppose, by contradiction, that $C \neq N$. Now let $L / N$ be a chief factor of $G$ with $N \leq L \leq C$. By the claim above, we deduce that $L / N$ is isomorphic to some nonabelian simple group. In particular, $|\rho(C / N)| \geq|\pi(L / N)| \geq 3$. We have that $M C / N \cong C / N \times M / N$. Since $\sigma(M C / N) \leq \sigma(M C) \leq \sigma(G) \leq 2$, we deduce that $\sigma(C / N \times M / N) \leq 2$ and thus by Lemma 3.1, $\sigma(C / N)=1=\sigma(M / N)$. By [12], we have $C / N \cong T \times A$, where $A$ is abelian, $T$ is a nonabelian simple group and $S, T \in\left\{\mathrm{PSL}_{2}(4), \mathrm{PSL}_{2}(8)\right\}$. Since $C \unlhd G$ and the solvable radical $W$ of $C$ is characteristic in $C$, we obtain that $W \unlhd G$, and thus $W \leq N$ as $N$ is the largest normal solvable subgroup of $G$. Clearly, $N \leq W$ as $N$ is also a solvable normal subgroup of $C$, so $W=N$. Therefore, $C / N$ has no nontrivial normal abelian subgroup. Thus, $A=1$, and hence $C / N \cong T$. Since $\pi(G / C)=\pi(M / N)$ and $G / N$ has no normal abelian Sylow subgroup, we obtain that

$$
\rho(G / N)=\pi(G / N)=\pi(C / N) \cup \pi(M / N)=\pi(S) \cup \pi(T) .
$$

It follows that

$$
|\rho(G / N)|=|\pi(S) \cup \pi(T)| \leq\left|\pi\left(\mathrm{PSL}_{2}(4)\right) \cup \pi\left(\mathrm{PSL}_{2}(8)\right)\right|=4 .
$$

Hence, $\rho(G)-\rho(G / N)$ is nonempty. Now let $r \in \rho(G)-\rho(G / N)$. As $\{2,3\} \subseteq \rho(G / N)$, we obtain that $r \notin\{2,3\}$. By Lemma 3.2, $r \in \rho(N)$, and hence $r \mid \theta(1)$ for some $\theta \in \operatorname{Irr}(N)$. Since $\sigma(M) \leq \sigma(G) \leq 2$ and $M / N \cong S$, by [19, Lemma 4.2], we deduce that $\theta$ extends to $\theta_{0} \in \operatorname{Irr}(M)$. Now let $\lambda \in \operatorname{Irr}(M / N)$ with $2 \mid \lambda(1)$. By Gallagher's theorem, $\varphi=\theta_{0} \lambda \in \operatorname{Irr}(M)$ with $\pi(\varphi(1))=\{2, r\}$. Notice that $r \geq 5$ since $r \notin\{2,3\}$. Now let $K=M C \unlhd G$. Then $K / M \cong T$ and $\sigma(K) \leq 2$. Applying the same argument as above, we deduce that $\varphi$ extends to $\varphi_{0} \in \operatorname{Irr}(K)$. Clearly, $K / M \cong T$ has an irreducible character $\mu$ with $3 \mid \mu(1)$ and thus, by Gallagher's theorem again, $\psi=\varphi_{0} \mu \in \operatorname{Irr}(K)$ and obviously $\sigma(\psi) \geq 3$, which is a contradiction.

We are now ready to prove Theorem B, which we state here. 
Theorem 3.4. Let $G$ be a group. If $\sigma(G) \leq 2$, then $|\rho(G)| \leq 2 \sigma(G)+1$.

Proof. Let $G$ be a counterexample to the theorem with minimal order. Then $\sigma(G) \leq 2$, but $|\rho(G)|>2 \sigma(G)+1$. If $G$ is solvable or $G$ is nonsolvable with $\sigma(G)=1$, then

$$
|\rho(G)| \leq 2 \sigma(G)+1
$$

by $[6,11,12]$, which is a contradiction. Thus, we can assume that $G$ is nonsolvable, $\sigma(G)=2$ and $|\rho(G)| \geq 6$. Let $N$ be the solvable radical of $G$. By Lemma 3.3, $G / N$ is an almost simple group with simple socle $M / N$. Since $\sigma(M / N) \leq \sigma(G / N) \leq \sigma(G)=2$, we deduce from Lemmas 2.5 and 2.4 that

$$
|\pi(G / N)|=|\pi(M / N)| \leq 5 .
$$

As $|\rho(G)| \geq 6$, we have that $\rho(G)-\rho(G / N)$ is nonempty and let $r \in \rho(G)-\rho(G / N)$. By Lemma 3.2, $r \mid \theta(1)$ for some $\theta \in \operatorname{Irr}(N)$. Since $\sigma(M) \leq 2$, by applying [19, Lemma 4.2], we deduce that $\theta$ extends to $\theta_{0} \in \operatorname{Irr}(M)$. Using Gallagher's theorem, we must have that $\sigma(M / N)=1$, and hence $M / N \cong \mathrm{PSL}_{2}(4)$ or $\mathrm{PSL}_{2}(8)$. Thus, $|\pi(G / N)|=|\pi(M / N)|=3$; hence, $|\tau| \geq 3$ with $\tau=\rho(G)-\rho(G / N)$. By Lemma 3.2, we have that $\tau \subseteq \rho(N)$ and, since $N$ is solvable, by applying Pálfy's condition [16, Theorem], there exists $\psi \in \operatorname{Irr}(N)$ such that $\psi(1)$ is divisible by two distinct primes in $\tau$. Now, by applying [19, Lemma 4.2] again, we obtain a contradiction. This contradiction shows that $|\rho(G)| \leq 2 \sigma(G)+1$, as wanted.

Acknowledgments. The author is grateful to the referee for a careful reading of the manuscript and for his or her corrections and suggestions.

\section{REFERENCES}

1. D. Alvis and M. Barry, Character degrees of simple groups, J. Alg. 140 (1991), $116-123$.

2. M. Barry and M. Ward, On a conjecture of Alvis, J. Alg. 294 (2005), 136-155.

3. C. Casolo and S. Dolfi, Prime divisors of irreducible character degrees and of conjugacy class sizes in finite groups, J. Group Theor. 10 (2007), 571-583.

4. J.H. Conway, R.T. Curtis, S.P. Norton, R.A. Parker and R.A. Wilson, Atlas of finite groups, Oxford University Press, Eynsham, 1985. 
5. H. Enomoto, The characters of the finite symplectic group $\operatorname{Sp}(4, q), q=2^{f}$, Osaka J. Math. 9 (1972), 75-94.

6. D. Gluck, A conjecture about character degrees of solvable groups, J. Alg. 140 (1991), 26-35.

7. B. Huppert, Research in representation theory at Mainz (1984-1990), in Representation theory of finite groups and finite-dimensional algebras, Progr. Math. 95, Birkhauser, Basel, 1991.

8. B. Huppert and W. Lempken, Simple groups of order divisible by at most four primes, Proc. F. Scorina Gomel State Univ. 16 (2000), 64-75.

9. I.M. Isaacs, Character theory of finite groups, AMS Chelsea Publishing, American Mathematical Society, Providence, Rhode Island, 2006.

10. G. Malle, Almost irreducible tensor squares, Comm. Alg. 27 (1999), 10331051.

11. O. Manz, Endliche auflösbare Gruppen, deren sämtliche Charaktergrade Primzahlpotenzen sind, J. Alg. 94 (1985), 211-255.

12. , Endliche nicht-auflösbare Gruppen, deren sämtliche Charaktergrade Primzahlpotenzen sind, J. Alg. 96 (1985), 114-119.

13. O. Manz and T. Wolf, Arithmetically long orbits of solvable linear groups, Illin. J. Math. 37 (1993), 652-665.

14. G.O. Michler, A finite simple group of Lie type has p-blocks with different defects, $p \neq 2$, J. Alg. 104 (1986), 220-230.

15. A. Moretó and P.H. Tiep, Prime divisors of character degrees, J. Group Theor. 11 (2008), 341-356.

16. P. Pálfy, On the character degree graph of solvable groups I, Three primes, Period. Math. Hungar. 36 (1998), 61-65.

17. W. Simpson and J. Frame, The character tables for $\operatorname{SL}(3, q), \operatorname{SU}\left(3, q^{2}\right)$, $\operatorname{PSL}(3, q), \operatorname{PSU}\left(3, q^{2}\right)$, Canad. J. Math. 25 (1973), 486-494.

18. B. Srinivasan, The characters of the finite symplectic group $\operatorname{Sp}(4, q)$, Trans. Amer. Math. Soc. 131 (1968), 488-525.

19. H.P. Tong-Viet, Groups whose prime graphs have no triangles, J. Alg. 378 (2013), 196-206.

20. D. White, Character degrees of extensions of $\mathrm{PSL}_{2}(q)$ and $\mathrm{SL}_{2}(q)$, J. Group Theor. 16 (2013), 1-33.

21. K. Zsigmondy, Zur Theorie der Potenzreste, Monatsh. Math. Phys. 3 (1892), 265-284.

Department of Mathematical Sciences, Kent State University, Kent, OH 44242

Email address: htongvie@kent.edu 